\title{
A TERRESTRIAL LASER SCANNING MEASUREMENT STATION TO MONITOR LONG-TERM STRUCTURAL DYNAMICS IN A BOREAL FOREST.
}

\author{
Mariana Campos ${ }^{1, *}$, Paula Litkey ${ }^{1}$, Yunsheng Wang ${ }^{1}$, Yuwei Chen ${ }^{1}$, Heikki Hyyti ${ }^{1}$, Juha Hyyppä ${ }^{1}$. Eetu Puttonen ${ }^{1}$ \\ 1 Department of Remote Sensing and Photogrammetry, Finnish Geospatial Research Institute (FGI) in the National Land Survey of \\ Finland, Geodeetinrinne 2, FI-02431 Masala, Finland - \\ (mariana.campos, paula.litkey, yunsheng.wang, yuwei.chen, heikki.hyyti, juha.hyyppa, eetu.puttonen)@ maanmittauslaitos.fi
}

KEY WORDS: TLS time series; vegetation phenology; plants dynamics; boreal forest monitoring.

\begin{abstract}
:
This work presents a data acquisition framework and the technical details of a permanent terrestrial laser scanning (TLS) measurement station for high spatial and temporal resolution forest observation that was developed in the Finnish Geospatial Research Institute. The TLS measurement station was established to provide hyper-temporal time series of three-dimensional point cloud data for long term monitoring of a boreal forest. Time series data acquisition framework consists of regular 14-minute scans performed by a RIEGL VZ2000i laser scanner in every 30 minutes, resulting in the collection of 48 scans per day. The entire framework includes the setting up of the laser scanner, the initialization of daily project, the scanning data acquisition over a preset time window, the storage management of the collected data at a local measurement computer, and the transfer of data from the measurement computer to network-attached storage (NAS) for further data processes. The operability of the proposed TLS measurement station was first piloted at a test area of about $32,500 \mathrm{~m}^{2}$ in Southern Finland $\left(60^{\circ} 09^{\prime} \mathrm{N}, 2^{\circ} 32^{\prime} \mathrm{E}\right)$. A set of several long monitoring experiments were performed over the whole growing season from the beginning of April to the end of October in 2019. As preliminary results, the time series outputs have captured detailed information on the phenological changes in the test site with sub-centimetre accuracy. For instance, it was possible to visualize plant dynamics phenomena, such as the sprouting of leaves in spring and their falling in autumn.
\end{abstract}

\section{INTRODUCTION}

The high penetrability of laser beam associated with the ability to acquire multiple returns per transmitted pulse makes the laser scanning systems a unique measurement tool for data acquisition (Wehr, Lohr, 1999), three-dimensional representation and biophysical analysis of forest environments (Eitel et al., 2016). Forest monitoring requires close-range information about trees to understand forest structure and internal dynamics (West et al., 2009). Terrestrial laser scanning (TLS) can acquire forest measurements from a close-to ground perspective and regardless the external lighting conditions, which enables the constant extraction of forest structural parameters at an individual tree level without interfering their internal processes. Therefore, the three-dimensional TLS data has an important role for ecological and economic forest analyses, such as mapping the horizontal and vertical arrangement of trees, tree competition, phenological changes, carbon balance, and nutrient-water cycle.

The capabilities of the TLS have been improved significantly in recent years due to device developments and the increase of computational capacity for data storage and processing. Forest monitoring and analyses that require sub-centimetre spatial and minute-level temporal resolutions are nowadays possible based on accurate three-dimensional laser point clouds. TLS has been widely used to detect the dynamic behaviour of a target within a time series data set, especially for building structure monitoring (e.g. architectural heritages, dams, railways and bridges). However, the high potential of TLS for long-term temporal phenomena monitoring of fully grown trees with high resolution is not yet fully explored. To fully utilize the information acquired with TLS-based time series, a rigorous and straightforward framework is required to detect and quantify target changes.

Controlled long and short-term vegetation monitoring in outdoor environment presents a significant technical challenge due to environmental factors like wind, temperature variation, and precipitation. Furthermore, high temporal-density scans and short scanning interval are needed to detect tree dynamic behaviour on a daily basis. This leads to large data sets that have to be processed and stored efficiently.

Here, we present a permanent TLS measurement station developed at the Finnish Geospatial Research Institute (FGI), aiming to monitor temporal phenomena in vegetation, such as these circadian and longer term structural dynamics. The TLS station was built to monitor boreal forest with high spatial and temporal resolution in a forest research station. The information acquired in the TLS station accurately detects structural changes in tree crowns. These changes can be linked with phenological state in plants and the internal processes driving the changes. This work presents the technical details in setting up the laser scanning measurement, the data acquisition and pre-processing workflows during a TLS monitoring pilot project.

\section{RELATED WORK}

Previous works have presented the potential of TLS data to detect and monitor vegetation phenology and seasonal dynamics as a function of time in forests and cultivated areas. The TLS time series temporal resolution depends on the monitored vegetation phenomena, which can be classified as long- or short-term timescale.

Long term seasonal phenology, such as spring sprouting and flowering (Calders et al., 2015), biomass change (Crommelinck, Höfle, 2016) and plant growth (Guo et al., 2019) can be monitored with repeating scans over weekly, monthly or annual intervals. Calders et al. (2015) provided insight about the feasibility of TLS measurements in the context of long term repeatable measurements for forest monitoring. A total of 48 scans days were performed in a deciduous forest in the

\footnotetext{
*mariana.campos@maanmittauslaitos.fi
} 
Netherlands between February and June 2014 to monitor changes in plant area index over time, showing TLS potential to study spring phenological changes. Crommelinck and Höfle (2016) monitored crop height and biomass changes of a maize field in Germany using a TLS time series collected with a low-cost static system. Eight scan acquisitions were performed between May and August 2015. Crop height models were generated to detect maize plant development with centimetre level accuracy. A similar study was performed by Guo et al. (2019) to monitor wheat crop growth from TLS time series. Scans were carried out over whole crop growth cycles between 2015-2016 and 20162017 in eastern China. In this work, experimental assessments with different multi-scan setup were performed to provide technical support for effective detection of plant height changes with centimetre accuracy.

Detection of short term physiological phenomena in plants, such as circadian rhythms and foliar nyctinasty, requires short timescale measurements with an hourly or even more frequent scanning repetition rate (Puttonen et al., 2015; Puttonen et al., 2016; Zlinszky et al., 2017; Herrero-Huerta et al., 2018; Puttonen et al. 2019). Circadian movements of birch trees branches and foliage were detected and quantified using TLS data in the study presented by Puttonen et al. (2016). TLS time series were acquired in two geographically different locations (Finland and Austria). The scanning interval was approximately 1 hour in Finland and 10 min in Austria, enabling the detection of a vertical movement between 5 and $10 \mathrm{~cm}$. Subsequently, Zlinszky et al. (2017) measured nocturnal changes of 22 different plant species over a 12-h period with repeating scan acquisitions, resulting in 18-scans TLS time series. These authors reported different types of circadian movements in the studied species. 2 $4 \mathrm{~h}$ cycles of minor crown movement were detected, with movements of around $1 \mathrm{~cm}$. Herrero-Huerta et al. (2018) set up a controlled environment experiment to detected leaf movement of two Calathea roseopicta plants. Scans were taken in an indoor environment over two days. Measurements during the first day were performed in natural lighting conditions and measurements in the second day were taken in complete darkness. Temperature, relative humidity and light conditions were measured continuously. Leafs displacements and volume variation were detected. Recently, Puttonen et al. (2019) monitored circadian rhythms in two Norway maples overnight using three TLS systems mounted on tripods. They collected data collection from sunset to sunrise every $20 \mathrm{~min}$, resulting in 130 scans. Circadian movements up to $10 \mathrm{~cm}$ were identified on branch tips.

These works highlight the capability of TLS data to transcend basic 3-D mapping information, enabling the monitoring of temporal changes of both spectral and spatial targets properties. However, the major part of studies were performed within limited time periods. Many challenges still remain in determining the best data management practises for the large datasets produced and how to quantify structural dynamics in tree canopies and branches. The permanent TLS measurement station presented in this work is developed to provide time series data for short and long-term analyses, which can provide new insights about boreal forest dynamics.

\section{TLS MEASUREMENT STATION}

\subsection{Settings}

The TLS station operability was first piloted in a set of several long monitoring experiments that were performed in a test area of about $227 \mathrm{~m} \times 143 \mathrm{~m}$ in Southern Finland $\left(60^{\circ} 09^{\prime} \mathrm{N}, 24^{\circ} 32^{\prime} \mathrm{E}\right)$. The measurement station includes a RIEGL VZ-2000i laser scanner installed in weather protected hood (RIEGL PHA200/1000) and a buffer computer that stored daily data acquisitions and transferred them to network storage as a batch process. The RIEGL VZ-2000i laser distance measurement is performed by the high-precision time-of-flight (ToF) measurement mode. Technical properties of RIEGL VZ-2000i are presented in Table 1 . The scanner can have laser pulse repetition rate of up to $1200 \mathrm{kHz}$ and natural targets.

\begin{tabular}{|l|c|}
\hline Scanner & RIEGL VZ-2000i \\
\hline Type/ Laser class & ToF/ Class 1A \\
Wavelength & $1550 \mathrm{~nm}$ \\
Scanning mechanism & vertically rotating multi-facet \\
& mirror, horizontally rotating head \\
Max. FOV & $100 / 360$ (vertical / horizontal) \\
Max. Scan frequency & $1200 \mathrm{kHz}$ \\
Range & $600 \mathrm{~m}^{1} / 1200 \mathrm{kHz}$ \\
Dist. accuracy/precision & $5 \mathrm{~mm} / 3 \mathrm{~mm}$ \\
Beam divergence $(\mathrm{rad})$ & $0.27 \mathrm{mrad} / 100 \mathrm{~m}$ \\
\hline
\end{tabular}

Table 1. Technical properties of RIEGL VZ-2000i

The measurement settings for the time series acquisition in this work were $1200 \mathrm{kHz}$ frequency with a detection range between 1 and $290 \mathrm{~m}$. The measurement scanning window was set vertically between $30^{\circ}$ and $100^{\circ}$ from the zenith and horizontally between $88^{\circ}$ and $222^{\circ}$ in counter-clockwise direction. The angular resolution in vertical and horizontal directions was set to $0.006^{\circ}$, which corresponds to about $1 \mathrm{~cm}$ horizontal and vertical point spacing at 100 -meter distance from the scanner. This fixed measurement configuration was used to scan the same scene and targets (trees and understory vegetation) within the test area. Each scan takes about 14 minutes, which produces $\sim 300$ million points per scan. Figure 1 shows the RIEGL VZ-2000i laser scanner facing the test area (Feb. /2020).

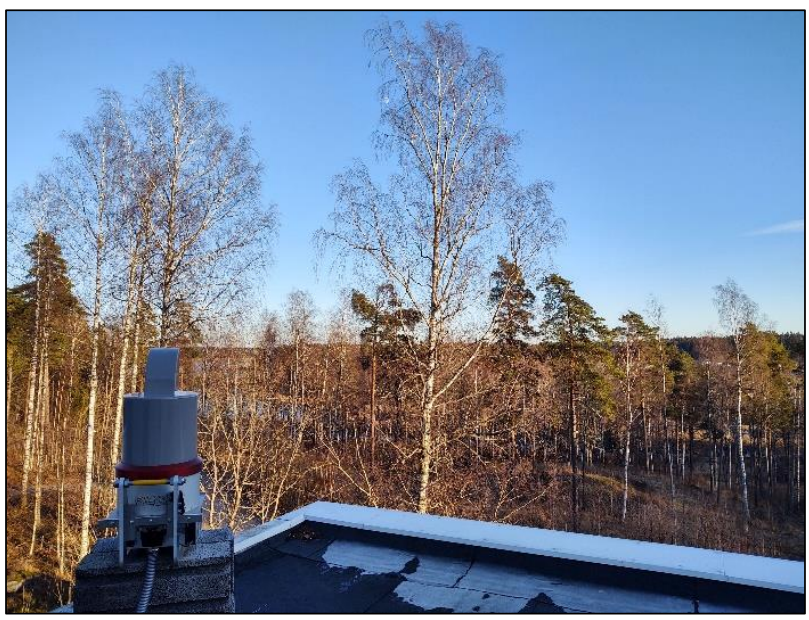

Figure 1. Test area view after data acquisition campaign in February 2020 with RIEGL VZ-2000i laser scanner facing the test area.

\footnotetext{
${ }^{1}$ Typical values for average conditions: flat targets with size in excess of the laser beam diameter, perpendicular angle of incidence, and atmospheric visibility of $23 \mathrm{~km}$.
} 


\subsection{Data Acquisition}

The time series data collection workflow consisted of a regular 24 hours interval and it was fully automated using python and shell scripts. The workflow included laser scanning set up, daily project initialization, scan acquisition over a preset time window, data local storage management, and data transfer from a measurement station unit computer to a network-attached storage (NAS) network-attached storage (NAS) located in FGI network.

Figure 2 summarizes the data acquisition workflow. A new data collection project is initialized every day. The 14-minute scanning were performed every 30 minutes, resulting in 48 scans per daily project. The TLS data was first stored in the measurement computer at the measurement station (MS). Each scan produced a point cloud file in proprietary RIEGL data format (.rxp) and related cover metadata ( $6 \mathrm{~GB}$ per scan). Once all 48 scans were collected, the complete project dataset was transferred overnight from the local measurement computer to the FGI-NAS ( 260 GB per day). Finally, if the data transfer was successful, the previous day project was to be deleted from the measurement computer.

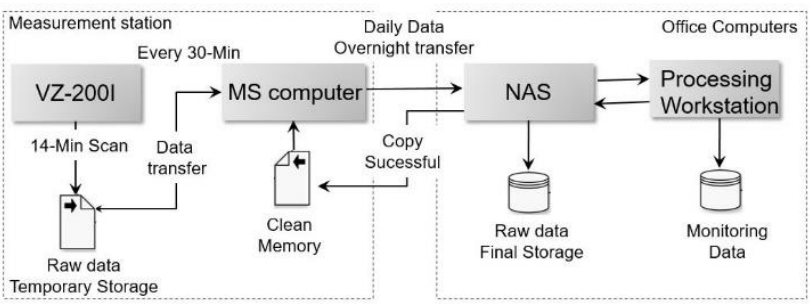

Figure 2. Data acquisition workflow of the TLS measurement station.

\subsection{Pre-processing and Output Data}

Datasets transferred to the NAS were systematically preprocessed in a separate workflow presented in Figure 3. The main workflow steps consist of .rxp to .laz file conversion, point cloud filtering, and co-registration of the time series (tiepoint search and registration).

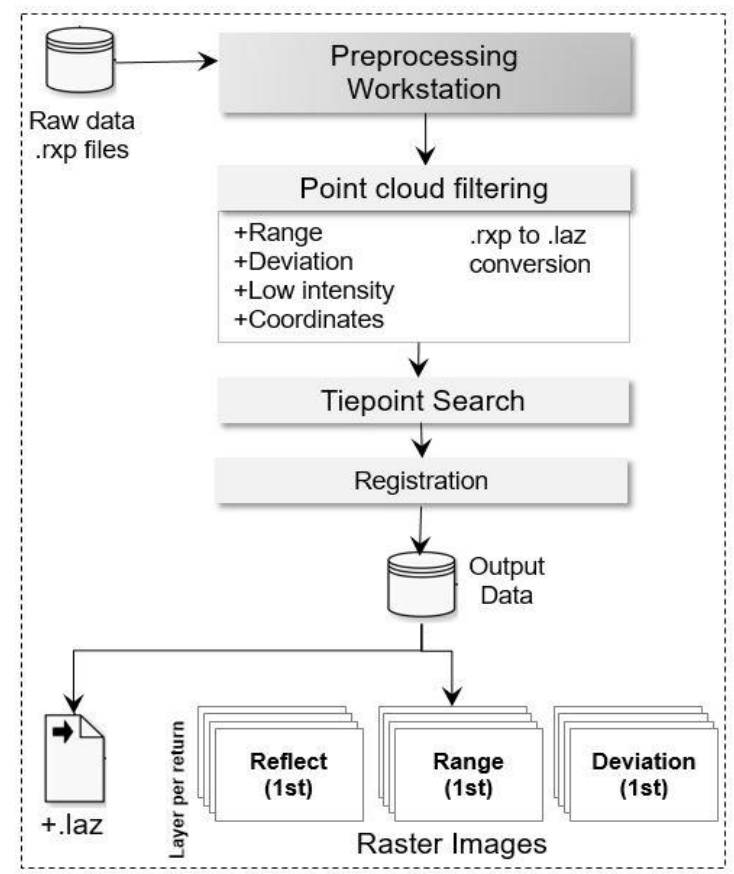

Figure 3. Preprocessing workflow and output data.
Raw scan data were pre-processed from .rxp format to compressed ASPRS .laz 1.4 format. The .rxp files are converted to .laz format using RivLib (version 7.1, RIEGL Gmbh, Austria) and LASzip (version 3.4, RapidLasso Gmbh, Germany) dynamic-link libraries (DLLs) combined in a $\mathrm{C} / \mathrm{C}++$ routine. The point cloud was filtered in the same routine. Range, deviation, low intensity and coordinates values were considered as a threshold to obtain a filtered point cloud. Instrumentation noise was excluded from the final laz files based on the range and scan angles filtering. Individual trees can be delineated by setting up a bounding box of the test area during the data conversion when focusing on individual tree point clouds analyses (Figure 4.b). The conversion workflow takes between 5 and 6 minutes per full scan over the measurement scene that consists of about 300 million points.

After conversion, the resulting point cloud in .laz for contains the filtered 3D coordinates $(\mathrm{X}, \mathrm{Y}, \mathrm{Z})$ of the points in a local reference system, point return number, number of returns, intensity, scan angles, reflectance, return pulse deviation and range (Figure 5). Additionally, separate range, reflectance and deviation raster images (.tiff) were produced of each scan for the first four laser return pulses (Figure $4 b$ to Figure $4 \mathrm{e}$ ). The $3 \mathrm{D}$ coordinates of the point cloud were used to generate the raster images via python scripts. The image coordinates of the points were computed using an equirectangular projection. Figure 4.a shows an example of a reflectance raster image. Figure 4.b to Figure 4.e exemplifies the four raster image layers of a range image from the first to the fourth return, respectively.

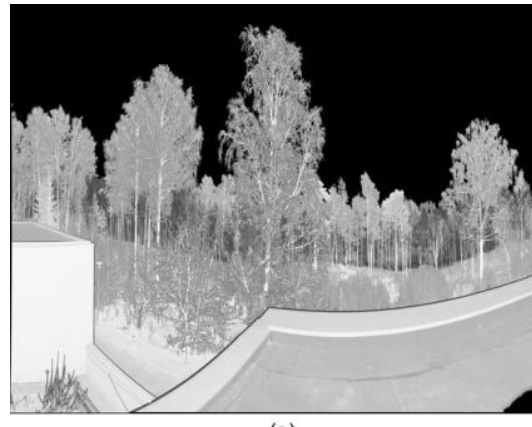

(a)

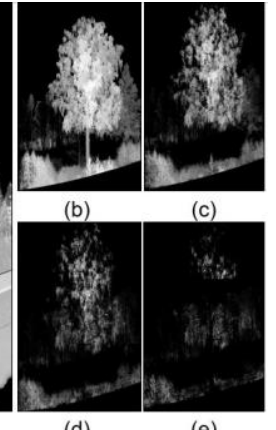

(d) (e)
Figure 4. Example of raster images, in which (a) show a reflectance image of the whole scanned scene. (b) - (e) present examples of raster image layers of a range image from the first to the fourth return, respectively.

The raster images provided a significant advantage because image processing techniques can be included in the workflow based on already existing information in collected TLS data, such as point range and reflectance values. Additionally, the point cloud mapping in rasters is carried out here without loss of resolution. Thus, the results of any operation can be transferred directly back to the other representation. For instance, automatic marker-free registration of the point cloud time series can be performed using keypoints from feature-based matching (FBM) methods, such as scale-invariant feature transform (SIFT) developed by Lowe (2004). The process includes image generation, histogram equalization, keypoint search, image matching, inlier detection and $3 \mathrm{D}$ rigid body transformation parameter estimation using the match points. Similar approached have been done by Alba et al. (2011), Houshiar et al. (2015) and Urban, Weinmann (2015). However, marker-free registration considering a forest scene is challenge, since trees are not targets having well-defined rigid shape and structure. A TLS point cloud registration method has been developed and it will be presented in details in future works. 


\section{PILOT PROJECT: PRELIMINARY RESULTS}

The pilot monitoring study was performed over the whole growing season from the beginning of April to the end of October in 2019. In total, this resulted in a large dataset consisting of about 10 thousand scans with the total size of 54 terabytes. Figure 5 shows an example of a point cloud acquired by the permanent TLS station in the middle April (18/04) at midnight. The point cloud was colourized considering the range parameter values, ranging from 1 to $290 \mathrm{~m}$.

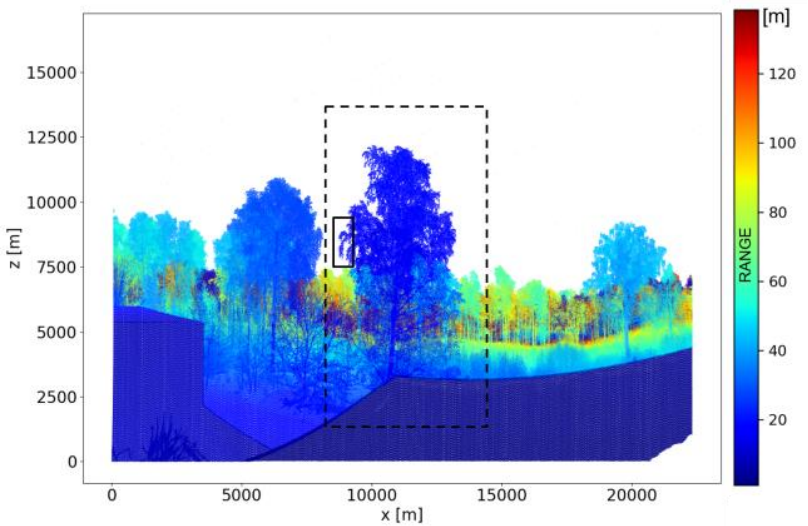

Figure 5. Example of point cloud colourized by range parameter values $(0-290 \mathrm{~m})$ acquired by the TLS measurement station during the pilot project (April). The dashed and solid bounding box show the position of the birch tree and its branch, respectively. Their phenological changes were studied with more detail.

Daily movement dynamics and seasonal changes can be detected in the TLS time series data. Therefore, a silver birch (Betula pendula) was selected in the dataset to demonstrate the phenological changes on two different temporal scales (daily and monthly). The positions of the Silver birch tree in the study area and the respective branch used in the experiments are highlighted in the Figure 5. These features were located at about $50 \mathrm{~m}$ away from the scanner. The silver birch tree was delineated from the complete point cloud for further analysis.

To demonstrate the diurnal movement detection in branches, an overnight dataset with no observed wind and without rainfall was selected. The scans in the dataset were acquired between 16/ May 8 PM and 17/May - 8 AM (26 scans). Temperature, precipitation and prevalent wind conditions were estimated by considering the 10 min-weather measurements of the closest weather station near the test area $\left(60.18^{\circ} \mathrm{N}, 24.79^{\circ} \mathrm{E}\right)$. The wind speed during the selected overnight was lower than $3 \mathrm{~m} / \mathrm{s}$, which means calm and light breeze in Beaufort scale rates. The precipitation intensity was $0 \mathrm{~mm} / \mathrm{h}$. The air temperature was ranging between 2 and 15 degrees Celsius. Circadian rhythm of the silver birch branch were monitored during the night using the clustering approach developed by Puttonen et al. (2019). Figure 6 show in (a) the clusters selected in the branch and (b) the displacement of the cluster centres from their initial location at $8 \mathrm{PM}$. On average, the clusters had moved around $5 \mathrm{~cm}$ from their initial locations after sunrise.
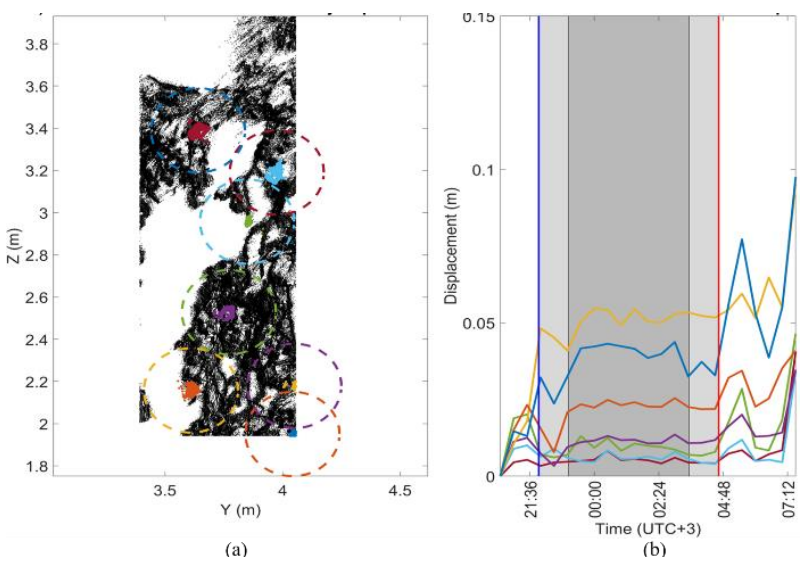

Figure 6. An example of overnight circadian rhythm monitoring in a Silver Birch branch between 16/ May - 8 PM to 17/May - 8

AM using the TLS measurement station data: (a) clusters selected in the branch and (b) the displacements of their centres from the initial locations, correspondingly

The seasonal phenology monitoring is demonstrated with the point clouds collected from the silver birch branch structure in four dates. The collection times were in middle spring (18/April), summer (18/June), autumn (18/Sept) and late autumn (11/Nov.) and their point clouds are presented in Figure 7. Visualization of the point clouds clearly shows the sprouting of leaves in spring and their falling in autumn. The all four days had similar wind speeds $(\sim 3 \mathrm{~m} / \mathrm{s})$ and precipitation intensities $(0 \mathrm{~mm} / \mathrm{h})$ at the time of their collection around midnight. The developed TLS station allows accurate monitoring and timing of spring phenology. This provides novel information to connect biological cycles with environmental conditions. For instance, systematic shifts in the beginning of the growing season in spring are a key phenological indicator of the effect of climate change in boreal forests, as discussed by Calders et al. (2015).

\section{CONCLUSIONS}

This study describes a pilot project using a permanent TLS measurement station to capture and monitor short- and long-term phenomena in vegetation. The TLS measurement station produces a high resolution spatial and temporal point cloud time series that provides detailed information on individual trees on hectare level, thus enabling non-disruptive capture of circadian rhythm dynamics and spring phenology changes in a natural forest environment. The weather conditions are a limitation and the scan data needs to be carefully curated to guarantee the point cloud quality when doing analysis. In this regard, the TLS measurement station was recently moved to a forest station, where the time series information will be linked with simultaneously collected meteorological and biophysical reference parameters for future works. 

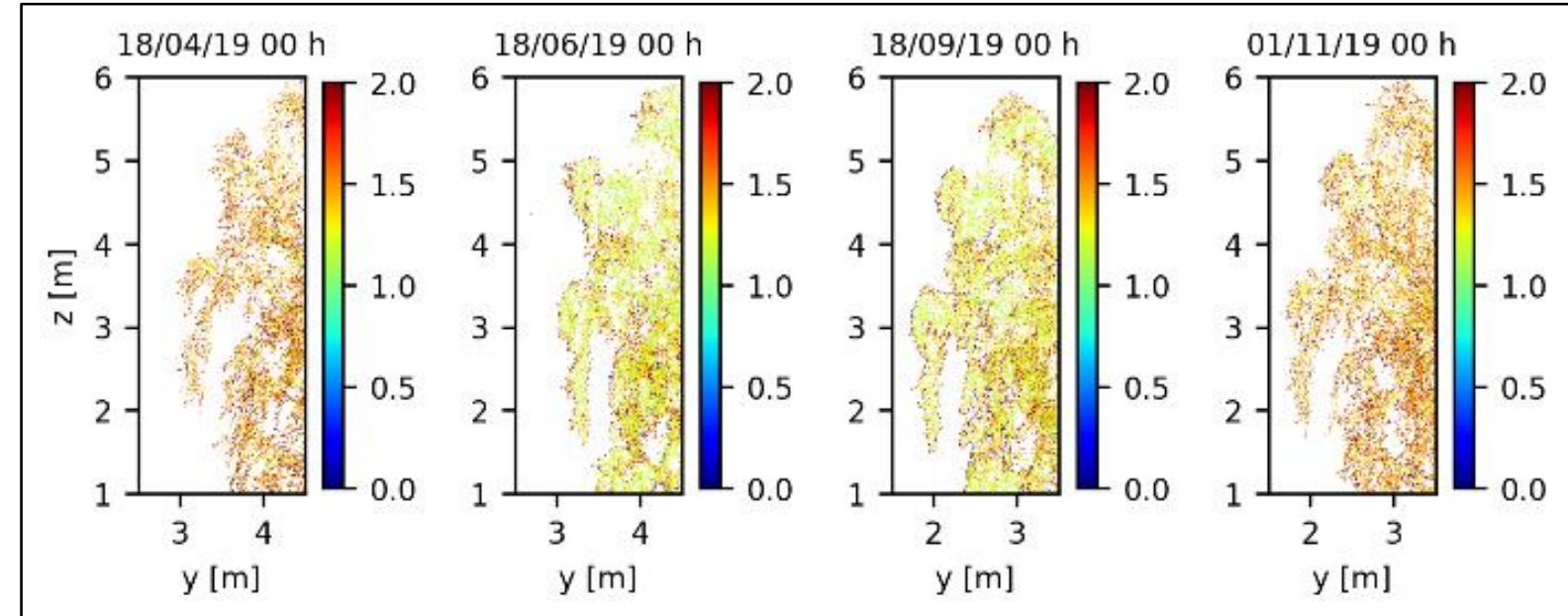

Figure 7. Seasonal variation in a Silver Birch branch measured with two to three month intevals between April and November in 2019. The colour scale shows point reflectance in logarithmic scale $(0-2.0)$.

\section{ACKNOWLEDGEMENTS}

The study received support from Academy of Finland projects no.316096/320075, "Upscaling of carbon intake and water balance models of individual trees to wider areas with short interval laser scanning time series" and from Strategic Research Council at the Academy of Finland project no. 293389/314312, "Competence Based Growth Through Integrated Disruptive Technologies of 3D Digitalization, Robotics, Geospatial Information and Image Processing/Computing - Point Cloud Ecosystem (COMBAT)".

\section{REFERENCES}

Alba, M., Barazzetti, L., Scaioni, M., Remondino, F., 2011: Automatic registration of multiple laser scans using panoramic rgb and intensity images. Int. Arch. Photogramm. Remote Sens. Spat. Inf. Sci, XXXVIII-5/W12, 29-31.

Calders, K., Schenkels, T., Bartholomeus, H., Armston, J., Verbesselt, J., Herold, M., 2015: Monitoring Spring Phenology With High Temporal Resolution Terrestrial Lidar Measurements. Agricultural and Forest Meteorology, 203, 158-168. doi: 10.1016/j.agrformet.2015.01.009

Crommelinck, S. C, Höfle, B.: 2016. Simulating An Autonomously Operating Low-Cost Static Terrestrial LIDAR For Multitemporal Maize Crop Height Measurements. Remote Sens. 8 (3), 205. doi: 10.3390/rs8030205

Eitel, J. U. H., Höfle, B., Vierling, L. A., Abellán, A., Asner, G. P., Deems, J. S., et al.: 2016. Beyond 3-D: The New Spectrum of Lidar Applications For Earth And Ecological Sciences. Remote Sens. Environ. 186, 372-392. doi: 10.1016/j.rse.2016.08.018

Guo, T., Fang, Y., Cheng, T., Tian, Y., Zhu, Y., Chen, Q., Qiu, X., Yao, X., 2019: Detection Of Wheat Height Using Optimized Multi-Scan Mode Of Lidar During The Entire Growth Stages. Computers and Electronics in Agriculture, 165, 104959.

Herrero-Huerta, M., Lindenbergh, R., Gard, W., 2018: Leaf Movements of Indoor Plants Monitored By Terrestrial LIDAR. Front. Plant Sci. 9, 189. doi: 10.3389/fpls.2018.00189
Houshiar, H., Elseberg, J., Borrmann, D., Nüchter, A., 2015: A Study of Projections For Key Point Based Registration Of Panoramic Terrestrial 3D Laser Scan. Geo-spatial Information Science, 18(1), 11-31. doi:10.1080/10095020.2015.1017913.

Puttonen, E., Hakala, T., Nevalainen, O., Kaasalainen, S., Krooks, A., Karjalainen, M., Anttila, K., 2015: Artificial target detection with a hyperspectral LiDAR over 26-h measurement. Optical Engineering, 54(1), 013105.

Puttonen, E., Briese, C., Mandlburger, G., Wieser, M., Pfennigbauer, M., Zlinszky, A., Pfeifer, N., 2016: Quantification of Overnight Movement Of Birch (Betula Pendula) Branches And Foliage With Short Interval Terrestrial Laser Scanning. Frontiers in plant science, 7, 222.

Puttonen, E., Lehtomäki, M., Litkey, P., Näsi, R., Feng, Z., Liang, X., Wittke, S., Pandžić, M., Hakala, M., Karjalainen, M., Pfeifer, N., 2019: A Clustering Framework for Monitoring Circadian Rhythm in Structural Dynamics in Plants From Terrestrial Laser Scanning Time Series. Front. Plant Sci., 10, 486. doi: 10.3389/fpls.2019.00486

Urban, S., Weinmann, M., 2015: Finding a good feature detectordescriptor combination for the 2D keypoint-based registration of TLS point clouds. ISPRS Annals of Photogrammetry, Remote Sensing \& Spatial Information Sciences, II-3/W5, 121-128. doi:10.5194/isprsannals-II-3-W5-121-2015.

Wehr, A., Lohr, U, 1999: Airborne Laser Scanning - An Introduction And Overview. ISPRS Journal of Photogrammetry and Remote Sensing, 54(2), 68-82. doi: 10.1016/S09242716(99)00011-8

West, P. W., 2009: Tree and forest measurement. New York: Springer.

Zlinszky, A., Molnár, B., and Barfod, A. S., 2017: Not All Trees Sleep the Same-High Temporal Resolution Terrestrial Laser Scanning Shows Differences in Nocturnal Plant Movement. Front. Plant Sci. 8,1814. doi: 10.3389/fpls.2017.01814 